\title{
Modélisation physique de pieux hélicoïdaux mis en place dans du sable
}

\section{DE HOLLANDA CAVALCANTI TSUHA N. AOKI}

Department of Geotechnics São Carlos School of Engineering University of São Paulo

Av. Trabalhador

Saocarlense, 400

São Carlos - SP - CEP 13566-590 Brazil

crishc@terra.com.br nelson,aoki@uol.com.br

\section{G. RAULT, L. THOREL}

Section mécanique des sols et centrifugeuse

Laboratoire Central des Ponts et Chaussées

BP 4129

44341 Bouguenais Cedex

France

Gerard.Rault@lcpc.fr Luc.Thorel@Icpc.fr

\section{J. GARNIER}

Division reconnaissance et mécanique des sols

Laboratoire Central des Ponts et Chaussées BP 4129

44341 Bouguenais Cedex France

Jacques.Garnier@lcpc.fr
Une relation théorique entre la charge d'arrachement et le couple nécessaire à la mise en place de pieux hélicoïdaux dans un massif de sable a été vérifiée expérimentalement en centrifugeuse. Des pieux modèles avec plusieurs configurations d'hélices (nombre et diamètre) ont été installés puis arrachés dans des massifs de sable reconstitués. Des essais de cisaillement d'interface ont été effectués afin de compléter cette recherche. La comparaison entre les résultats théoriques et les résultats expérimentaux est détaillée et montre un bon accord. On a aussi comparé les résultats obtenus par cette modélisation en centrifugeuse avec des résultats d'essais sur pieux en vraie grandeur mis en place dans un silt sableux, et les deux sont du même ordre de grandeur. En plus, pour identifier le mécanisme de rupture de ce type de fondation dans du sable, on a réalisé des essais d'arrachement sur des pieux modèles installés à $1 \mathrm{~g}$ dans un massif préparé avec des couches de sable colorées.

Mots-clés : modélisation physique, centrifugeuse, pieux hélicoïdaux, résistance à l'arrachement, couple d'installation, angle de frottement à l'interface, sable.

\section{Physical modelling of helical pile anchors installed in sand}

A theoretical relationship between the uplift capacity and the installation torque of helical piles anchors in sand was verified using centrifuge modelling. Model piles with different dimensions (number of helices and diameter) were tested (pile installation and pull-out tests) in reconstituted sand samples.

Direct shear interface tests were conducted to complement this research. A comparison between theoretical and experimental results is presented, and the results show good agreement. Also, the results of this centrifuge modelling were compared to the test results on full-scale piles installed in sandy silt, and both have the same order of magnitude. In addition, pull-out tests on piles installed at $1 \mathrm{~g}$ in container prepared with layers of colored sand were conducted to identify the mechanism of rupture of this type of foundation in sand.

Key words: physical modelling, centrifuge, helical pile, uplift capacity, installation torque, interface friction angle, sand. 


\section{Introduction}

Les pieux hélicoïdaux sont utilisés comme fondations pour les tours et les pylônes autoportants ou haubanés. Ils sont constitués d'une tige métallique de section circulaire avec hélice soudée (une ou plusieurs). Le diamètre des hélices est choisi en considérant la capacité portante du sol et la charge de l'ouvrage à supporter. Ce type de pieu est installé par rotation dans le sol (vissage) à l'aide de moteurs hydrauliques montés sur un équipement léger. Des corrélations empiriques entre le couple appliqué pendant l'installation et la capacité portante du pieu sont habituellement utilisées pour contrôler sa mise en place. Il est enfoncé jusqu'à ce que la valeur du couple appliqué ait atteint un seuil particulier. Différentes relations théoriques entre la charge d'arrachement et le couple nécessaire pour le fonçage des pieux vissés ont été rapportés dans la littérature géotechnique (Narasimha Rao et al., 1989; Ghaly et al., 1991; Ghaly et Hanna, 1991 ; Perko, 2000).

Tsuha (2007) a proposé une méthode théorique simplifiée pour estimer la charge d'arrachement des pieux hélicoïdaux dans du sable à partir du couple mesuré à la fin de l'installation. Une approche expérimentale basée sur la modélisation physique en centrifugeuse au LCPC (Laboratoire Central des Ponts et Chaussées) à Nantes, a apporté un ensemble de résultats qui permettent de valider cette relation. Les modèles réduits de pieux hélicoïdaux ont été installés dans les massifs de sable reconstitués à deux densités différentes. Les essais de chargement ont été opérés dès la fin de la mise en place des pieux. Comme le couple appliqué au cours de l'installation du pieu dépend de la résistance de frottement entre l'hélice et le sol environnant, des essais de cisaillement d'interface caractérisant le contact entre la lame d'hélice et le sable ont été réalisés pour compléter ce travail.

Pour vérifier cette modélisation en centrifugeuse, on a comparé les résultats avec ceux obtenus sur pieux en vraie grandeur, semblables aux modèles utilisés pour cette investigation.

De plus, pour observer qualitativement la surface de rupture dans le massif de sable après l'arrachement des pieux hélicoïdaux, on a réalisé un conteneur préparé avec des couches de sable colorées (sable de même nature coloré avec une pigmentation bleue) intercalées régulièrement entre des couches de sable blanc.

\section{Relation théorique}

La vérification de la relation proposée par Tsuha (2007) entre le couple et la charge d'arrachement correspondant aux hélices des pieux hélicoïdaux dans un massif de sable, s'appuie sur la géométrie de la figure 1 qui montre la distribution du couple d'installation et de la charge d'arrachement du pieu hélicoïdal avec trois hélices. par :

La charge d'arrachement $Q_{u}$ (Fig. 1) est définie

$$
Q_{u}=Q_{h}+Q_{s}
$$

où $Q_{u}$ est la charge d'arrachement; $Q_{h^{\prime}}$ la charge d'arrachement relative aux hélices et $\mathrm{Q}_{\mathrm{s}^{\prime}}$ la résistance par frottement latéral sur la tige du pieu.

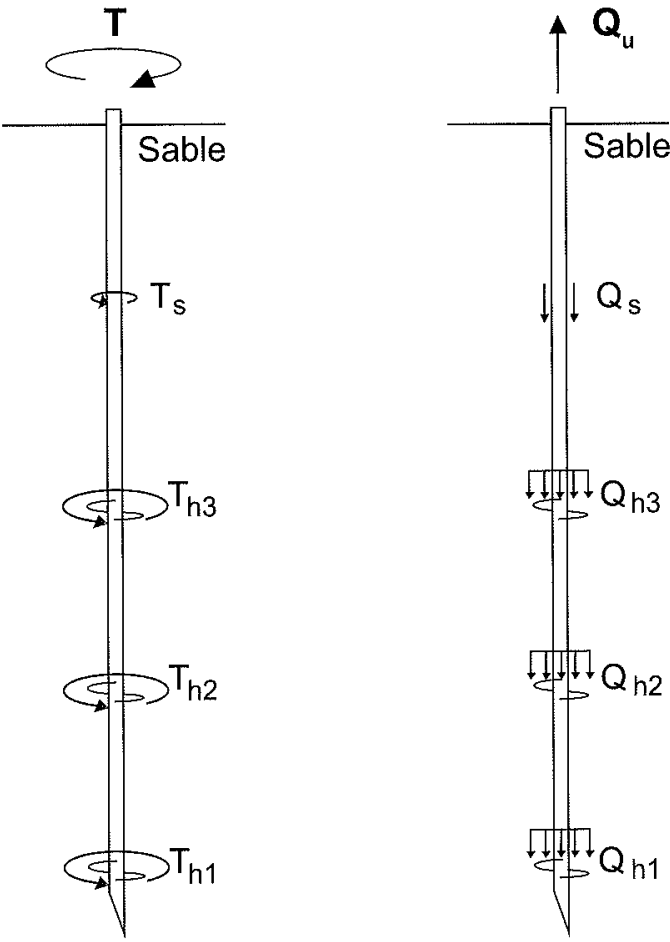

Ho. 1 Distribution du couple d'installation et de la charge d'arrachement du pieu.

Distribution of pile installation torque and uplift forces.

La charge d'arrachement relative aux hélices $Q_{h}$ est décrite par:

$$
Q_{h}=\sum_{i=1}^{N} Q_{h i}
$$

où $Q_{h i}$ représente la charge d'arrachement relative à l'hélice $\mathrm{i}$, indice variant de 1 à $N$, et $N$, le nombre d'hélices.

Le couple nécessaire à l'installation des pieux hélicoïdaux T est donné par :

$$
\mathrm{T}=\mathrm{T}_{\mathrm{h}}+\mathrm{T}_{\mathrm{s}}
$$

où T est le couple d'installation; $T_{h}$, le couple d'installation repris par les hélices et $\mathrm{T}_{\mathrm{s}^{\prime}}$ le couple d'installation repris par frottement latéral.

La part du couple d'installation reprise par les hélices $\mathrm{T}_{\mathrm{h}}$ est définie par :

$$
T_{h}=\sum_{i=1}^{N} T_{h i}
$$

où $\mathrm{T}_{h i}$ désigne la portion du couple d'installation reprise par l'hélice i.

La relation proposée entre la charge d'arrachement relative aux hélices $Q_{\mathrm{h}}$ et le couple d'installation repris par les hélices $T_{n^{\prime}}$ à la fin de la mise en place des pieux vissés dans un massif de sable, est fondée sur le mécanisme physique fréquemment utilisé pour une vis de transmission (trouvée dans la littérature relative au calcul d'éléments de machines) mais adapté aux pieux hélicoïdaux. Dans cet arrangement (Fig. 2), l’hélice du pieu est équivalente à une hélice de la vis (filet), le sable à l'écrou, l'angle de frottement entre l'hélice du pieu et le sable voisin $\delta_{r}$ à l'angle de frottement entre la vis et l'écrou, la charge à déplacer (W) à la charge d'arrachement relative aux hélices $\left(Q_{h}\right)$, et le couple nécessaire pour tourner la vis contre la charge (T) au couple d'installation repris par les hélices $\left(\mathrm{T}_{\mathrm{h}}\right)$. 

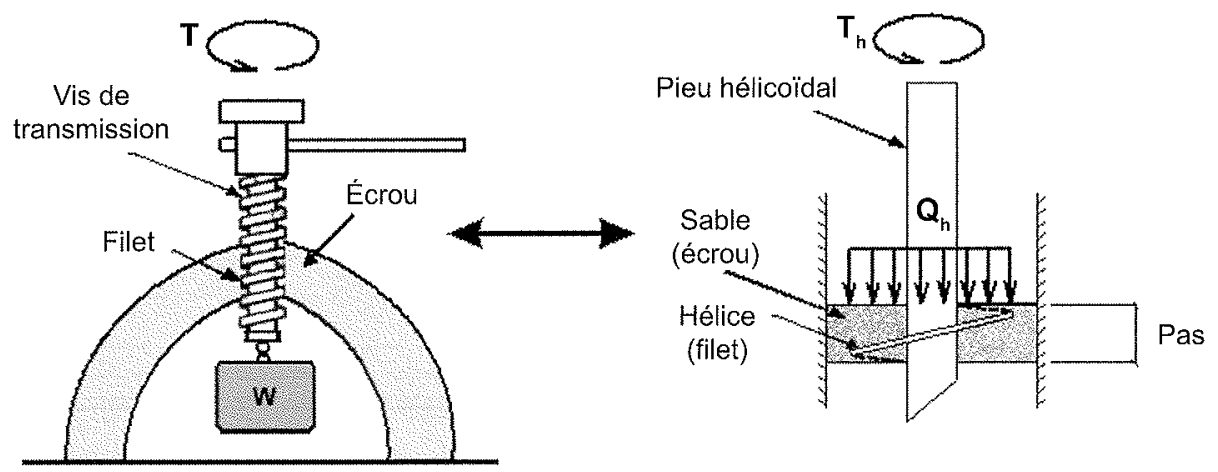

HG. 2 . Adaptation du mécanisme de la vis de transmission au pieu hélicoïdal dans du sable. Adjustement of the power screw mechanism to helical pile.
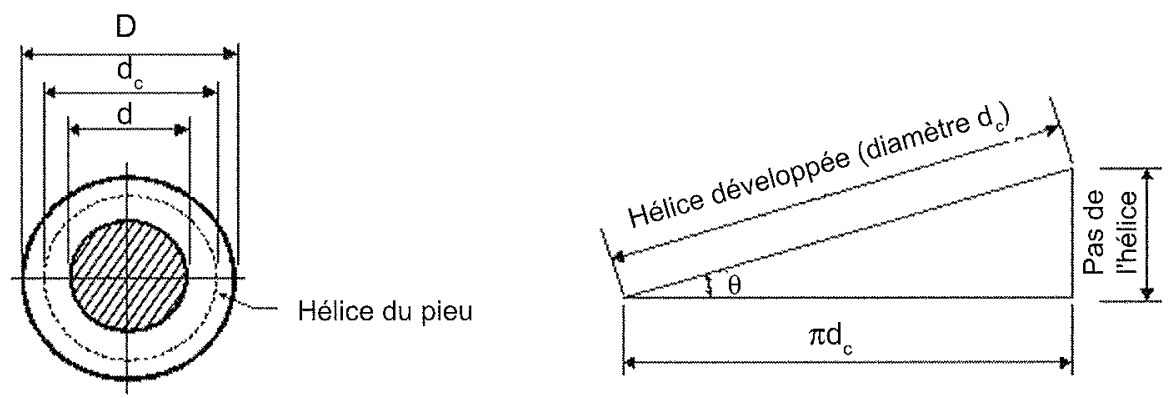

n6.3 Hélice du pieu hélicoïdal.

Pile helical plate.

L'équation obtenue à partir du mécanisme montré à la figure 2, pour déterminer la charge d'arrachement relative aux hélices $Q_{h}$ à partir du couple d'installation repris par les hélices $T_{n}$, est la suivante:

$$
Q_{h}=\frac{2 T_{h}}{d_{c} \cdot \operatorname{tg}\left(\theta+\delta_{r}\right)}
$$

où $\mathrm{d}_{\mathrm{c}}$ est le diamètre du cercle correspondant à la surface de l'hélice où les forces sont concentrées pendant l'installation du pieu (équation 6 et figure 2) ; $\theta$, l'angle de l'hélice formé avec l'horizontale au diamètre $\mathrm{d}_{c}$ (équation 7 et figure 3 ) et $\delta_{r}$ l'angle de frottement résiduel à l'interface entre l'hélice et le sable :

$$
\begin{gathered}
d_{c}=\frac{2}{3}\left\lceil\frac{D^{3}-d^{3}}{D^{2}-d^{2}}\right\rceil \\
\theta=\tan ^{-1}\left(\frac{p}{\pi d_{c}}\right)
\end{gathered}
$$

où $\mathrm{D}$ est le diamètre de l'hélice; $\mathrm{d}$, le diamètre de la tige et $\mathrm{p}$, le pas de l'hélice au diamètre $\mathrm{d}_{\mathrm{c}}$.
3

\section{Validation expérimentale au moyen d'un modèle centrifugé}

\author{
5.
}

\section{Dispositif expérimental}

La modélisation physique en centrifugeuse est une alternative expérimentale qui permet la réalisation d'essais sur différents modèles de pieux hélicoïdaux dans le même massif (dont les propriétés sont connues). Ces essais ont pour justification première de recréer au sein du corps d'épreuve, qui sera, si possible, construit à chaque fois à partir des mêmes matériaux que le prototype, le même champ de forces de masse que dans l'ouvrage en vraie grandeur (Corté, 1989). Des relations de similitudes permettent de relier le modèle réduit à un ouvrage en vraie grandeur ( prototype » correspondant (Garnier et al., 2007).

Les essais ont été réalisés sur la centrifugeuse du LCPC (Garnier et al., 1999).

Trois types de modèles réduits de pieux ont été réalisés avec ou sans hélices (Fig. 4) de façon à estimer la part du couple et de la charge reprise par les hélices seules. Les modèles de pieux hélicoïdaux sont composés d'une ou plusieurs hélices soudées sur une même 

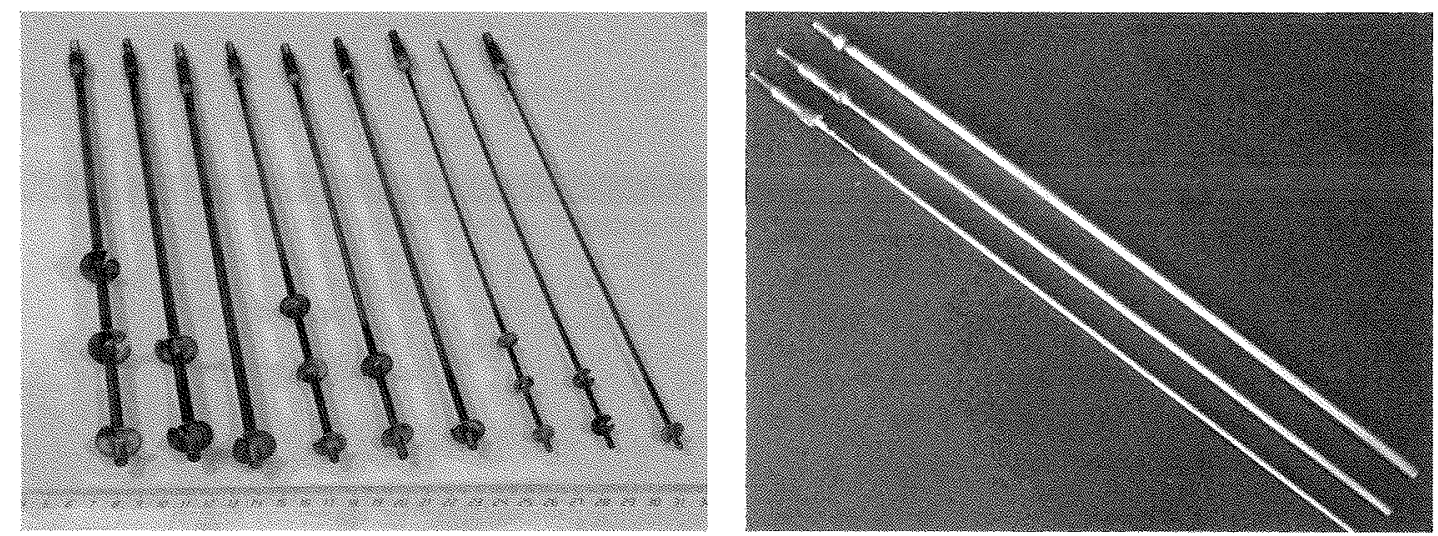

\begin{tabular}{|c|c|c|c|c|c|c|}
\hline \multicolumn{7}{|c|}{ FlG. 4} \\
\hline TABLEAUI I & \multicolumn{6}{|c|}{$\begin{array}{l}\text { Dimensions des pieux modèles }(\mathrm{M}) \text { et prototype }(\mathrm{P}) \text {. } \\
\text { Model }(\mathrm{M}) \text { and prototype }(\mathrm{P}) \text { pile dimensions. }\end{array}$} \\
\hline $\begin{array}{l}\text { Mrovere } \\
\text { he prea }\end{array}$ & $\begin{array}{l}\text { Nombre } \\
\text { dhelles }\end{array}$ & 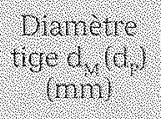 & 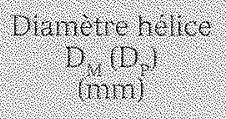 & $\begin{array}{l}\text { Pas de rhelice } \\
\text { p. (po) } \\
\text { (mm) }\end{array}$ & 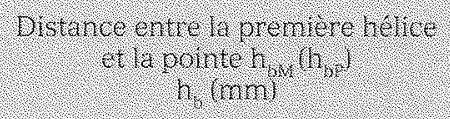 & Piche prototyje \\
\hline 1 & 1 & $3,0(64,3)$ & $10(214)$ & $3,0(64,3)$ & $10(214)$ & 3,1 \\
\hline 2 & 2 & $3,0(64,3)$ & $10(214)$ & $3,0(64,3)$ & $10(214)$ & 3,1 \\
\hline 3 & 3 & $3,0(64,3)$ & $10(214)$ & $3,0(64,3)$ & $10(214)$ & 3,1 \\
\hline 4 & 1 & $4,5(97,7)$ & $15(326)$ & $3,2(69,5)$ & $10(217)$ & 4,6 \\
\hline 5 & 2 & $4,5(97,7)$ & $15(326)$ & $3,2(69,5)$ & $10(217)$ & 4,6 \\
\hline 6 & 3 & $4,5(97,7)$ & $15(326)$ & $3,2(69,5)$ & $10(217)$ & 4,6 \\
\hline 7 & 1 & $6,0(132)$ & $20(440)$ & $3,5(77)$ & $10(220)$ & 6,2 \\
\hline 8 & 2 & $6,0(132)$ & $20(440)$ & $3,5(77)$ & $10(220)$ & 6,2 \\
\hline 9 & 3 & $6,0(132)$ & $20(440)$ & $3,5(77)$ & $10(220)$ & 6,2 \\
\hline 10 & - & $3,0(64,3)$ & - & - & - & 3,1 \\
\hline 11 & - & $4,5(97,7)$ & - & - & - & 4,6 \\
\hline 12 & - & $6,0(132)$ & - & - & - & 6,2 \\
\hline
\end{tabular}

Tableau II Propriétés du sable utilisé. Sand properties.

\begin{tabular}{|c|c|c|}
\hline Massil & Proprieted & Wrletr. \\
\hline Conteneur 1 & $\begin{array}{l}\text { Poids volumique } \\
\text { Indice de densité I } \\
\text { Angle de frottement }\end{array}$ & $\begin{array}{l}15,46 \mathrm{kN} / \mathrm{m}^{3} \\
56 \% \\
31 \text { degrés }\end{array}$ \\
\hline Conteneur 2 & $\begin{array}{l}\text { Poids volumique } \\
\text { Indice de densité } I_{D} \\
\text { Angle de frottement }\end{array}$ & $\begin{array}{l}16,30 \mathrm{kN} / \mathrm{m}^{3} \\
85 \% \\
41 \text { degrés }\end{array}$ \\
\hline
\end{tabular}

tige à intervalle de trois fois le diamètre de l'hélice. Quels que soient le nombre et le type d'hélice, tous les pieux modèles ont une longueur de $355 \mathrm{~mm}$. Douze modèles différents de pieux ont été réalisés (tableau I et figure 5). Compte tenu des fiches différentes selon le modèle de pieu, les échelles de réduction correspon- dant aux trois types de modèles sont 1/21,44, 1/21,71 et $1 / 22$

Les massifs ont été reconstitués avec du sable de Fontainebleau, par pluviation automatique dans l'air, dans des conteneurs rectangulaires rigides $(1200 \mathrm{~mm}$ $\times 800 \mathrm{~mm} \times 360 \mathrm{~mm})$. Deux densités différentes ont été retenues (Tableau $\mathrm{II}$ ).

\section{?}

\section{Procédure d'essais à la centrifugeuse}

Après installation du conteneur dans la centrifugeuse. chaque pieu modèle a été mis en place dans le sable, en vol à 22 g, au moyen du dispositif présenté (Figs. 6 et 7), par fonçage puis vissage. La vitesse de rotation de l'hélice reste constante à 5,7 tr/minute et la vitesse de fonçage est adaptée au pas du pieu hélice à mettre en place. Les profondeurs finales des pieux modèles installés dans le massif varient avec le diamètre de l'hélice (Fig. 5). Pendant le fonçage, les mesures de couple, déplacement, vitesse de fonçage et force sur les pieux 


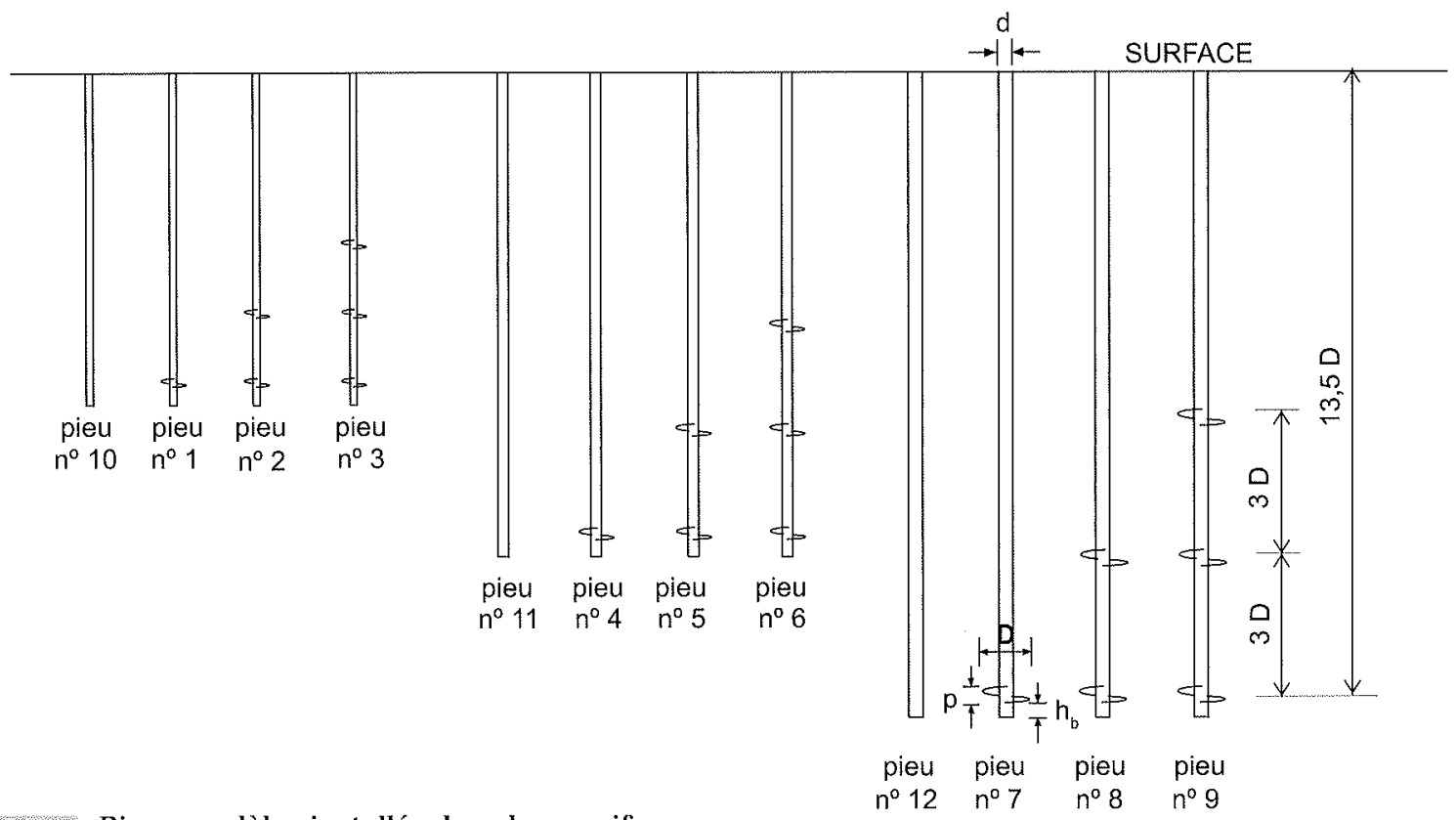

16. 5 Pieux modèles installés dans le massif.

Model piles embedded in the sand sample.

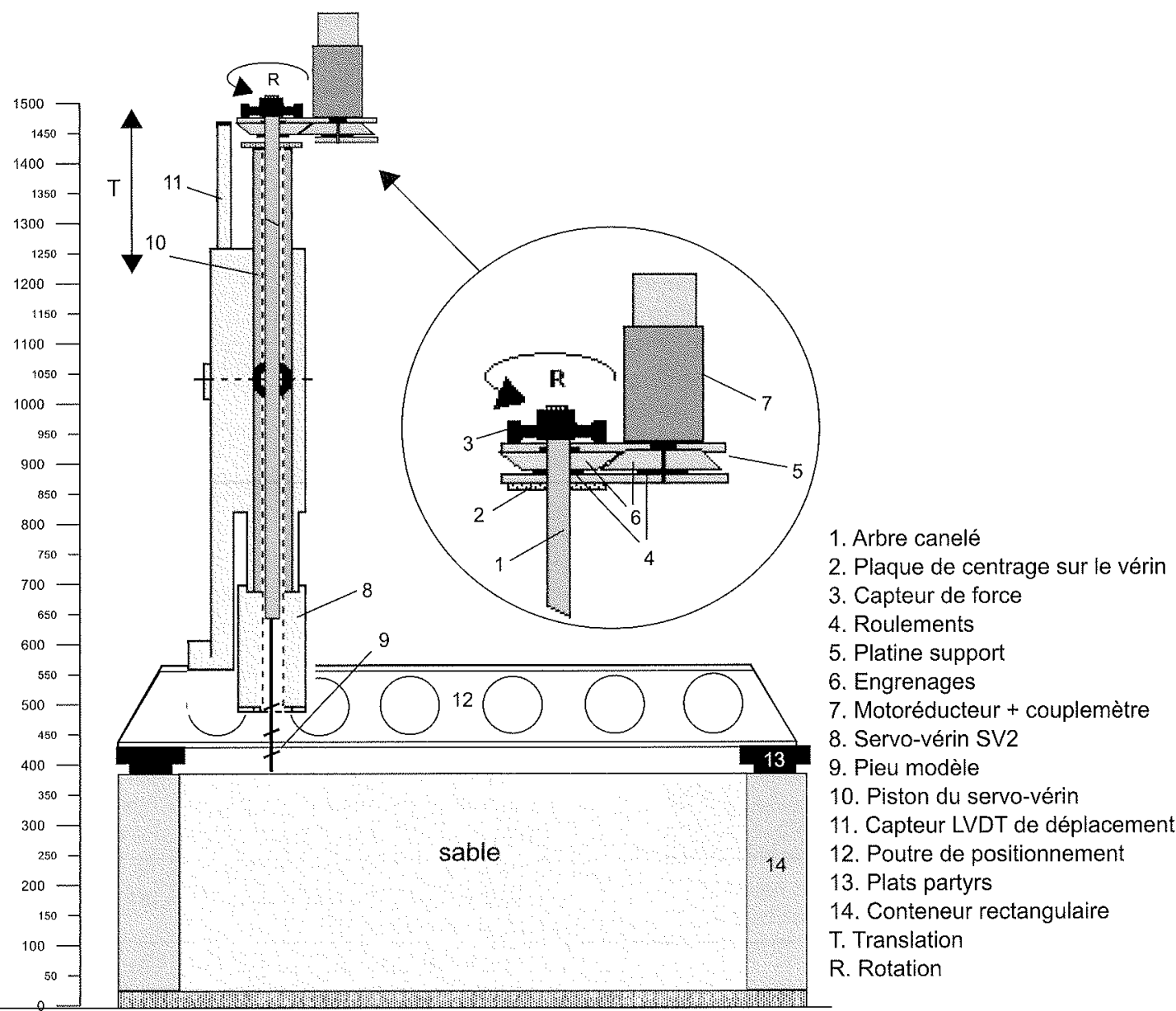

sont enregistrées par le système d'acquisition de données embarqué en centrifugeuse (Thorel et al., 2008).

Le chargement du pieu à hélice est opéré dès la fin de la mise en place. Une courte période d'attente est observée (1 minute) entre les phases de placement et d'arrachement afin de s'assurer de la relaxation d'efforts résiduels de fonçage. L'arrachement est alors enchaîné à vitesse constante $(1 \mathrm{~mm} / \mathrm{s})$. 

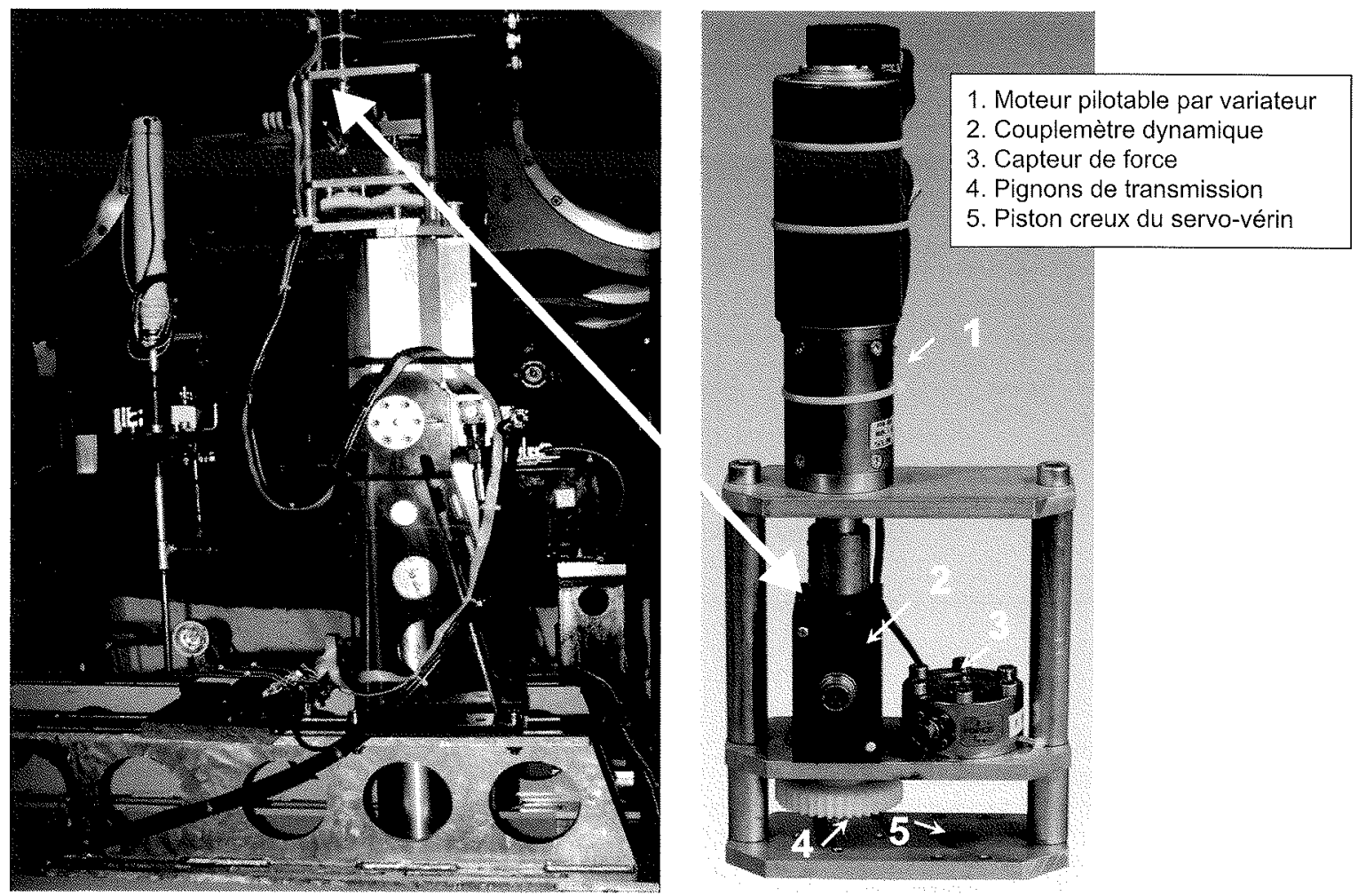

6. 7 Photographie du dispositif d'essai.

Photography of the test apparatus.

\section{8}

\section{Essais à la boîte de cisaillement}

Pour valider l'équation (5) du modèle théorique, des essais à la boîte de Casagrande ont été réalisés afin de déterminer l'angle de frottement d'interface $\delta$, entre la matière des hélices des pieux modèles et le sable utilisé pour la reconstitution des massifs lors des essais dans la centrifugeuse. Les hélices des pieux modèles (Fig. 8) sont des disques d'acier percés au diamètre de la tige centrale et soudés par brasure (soudure à base de cuivre-laiton) dont la surface représente $50 \%$ de la surface totale de l'hélice.

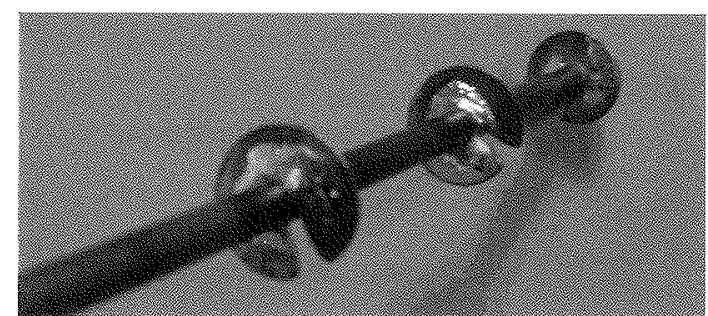

FIG. 8 Surface des hélices du pieu modèle. Helical plate surfaces of the model pile.

Les essais de cisaillement ont été réalisés alternativement avec des échantillons d'acier ou de laiton dans la demi-boîte inférieure. Les valeurs de $\delta_{r}$ obtenues pour chaque nature de matériau sont présentées dans le tableau III. Une valeur moyenne est calculée pour chaque valeur de densité sur la base de la répartition surfacique de la brasure.

La vérification de la formulation (5) utilise la valeur moyenne obtenue $\delta_{r}$ 27ableau il Angle de frottement résiduel à l'interface entre l'hélice et le sable.

Residual interface friction angle between helical plate material and sand samples.

\begin{tabular}{|c|c|c|}
\hline \multirow{2}{*}{ Winterface } & \multicolumn{2}{|c|}{$\begin{array}{l}\text { Angle de trortement resicuet at I interface o } \\
\text { (decres) }\end{array}$} \\
\hline & Sable $1 .=56 \%$ & $5661011=-86 \%$ \\
\hline Acier & 10,4 & 14,0 \\
\hline Laiton & 10,8 & 16,2 \\
\hline Moyenne & 10,6 & 15,1 \\
\hline
\end{tabular}

\section{Résultats et discussion}

Les résultats des essais en centrifugeuse convertis en prototype (vraie grandeur) sont présentés dans le tableau IV. Pour l'estimation du couple, on a retenu la valeur moyenne mesurée à la fin du vissage. Pour la charge d'arrachement, on a adopté la valeur de pic (valeur maximale).

Les valeurs de couple à la fin du fonçage et de la charge d'arrachement des pieux 10, 11, 12 sans hélice (Fig. 5) correspondent au frottement latéral sur les tiges des pieux 1 à 9 mobilisé pendant l'installation et l'arrachement des pieux $\left(T_{s}\right.$ et $\left.Q_{s}\right)$.

Les valeurs du couple d'installation repris par les hélices $\mathrm{T}_{h}$ et de la charge d'arrachement relative aux hélices $Q_{\text {}}$ ont été obtenues par la différence entre les résultats des essais des pieux avec hélices et des pieux sans hélice, avec les mêmes diamètres de tige et profondeur d'installation. 
La figure 9 (résultats convertis en prototype) montre les variations du couple nécessaire pour le fonçage des pieux 1, 2, 3 et 10 enregistrées lors de l'installation dans le massif de sable dense (conteneur 2).

La figure 10 permet d'estimer la part reprise par les hélices seules, déduction faite du frottement mesuré sur le fût du pieu sans hélice.

Les valeurs de $T_{n}$ (Tableau IV) et de $\delta_{r}$ (Tableau III) sont introduites dans l'équation (5) pour obtenir les valeurs de $\mathrm{Q}$, présentées dans le tableau IV. On constate que les résultats du couple d'installation repris par les hélices $T_{n}$ et de charge d'arrachement relative aux hélices $Q_{h}$ des pieux hélicoïdaux sont fortement influencés par la densité du massif.

La figure 11 montre la comparaison entre les valeurs calculées et les résultats expérimentaux de la charge d'arrachement relative aux hélices $Q_{h}$ (transformées en prototype). La résistance $Q_{n}$ calculée est obtenue par la relation (5) à partir du couple mesuré lors de l'installation des pieux. On observe une bonne concordance entre les valeurs théoriques et les résultats expérimentaux obtenus sur les modèles réduits testés en centrifugeuse.

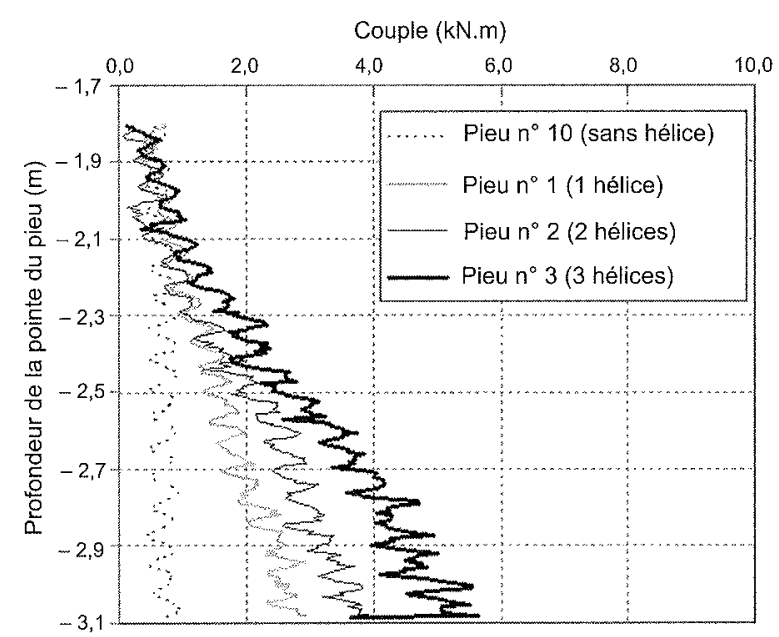

16.9 Exemple de couple mesuré pendant l'installation du pieu hélice en fonction de la profondeur (pieux modèles nos $1,2,3$ et 10 installés dans le conteneur 2 (valeurs en prototype)).

Example of the measured torque during helical pile installation versus depth (model piles nus 1,2 , 3 and 10 in the container 2 (prototype values)).

\begin{tabular}{|c|c|c|c|}
\hline TABLEAU W & \multicolumn{3}{|c|}{$\begin{array}{l}\text { Résultats des essais en centrifugeuse (convertis en prototype). } \\
\text { Centrifuge tests results (prototype values). }\end{array}$} \\
\hline Massif & Pieux nocteles & $\begin{array}{l}\text { fouple anstaliation repris } \\
\text { parles heles. }\end{array}$ & 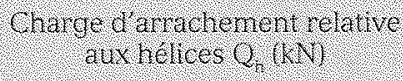 \\
\hline \multirow{9}{*}{ 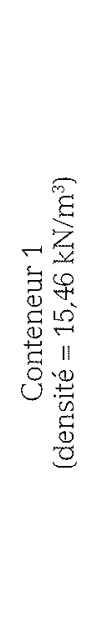 } & 1 & 0,3 & 14 \\
\hline & 2 & 0,4 & 19 \\
\hline & 3 & 1,0 & 43 \\
\hline & 4 & 1,6 & 46 \\
\hline & 5 & 3,2 & 83 \\
\hline & 6 & 3,3 & 112 \\
\hline & 7 & 4,1 & 69 \\
\hline & 8 & 4,9 & 108 \\
\hline & 9 & 5,3 & 150 \\
\hline \multirow{9}{*}{ 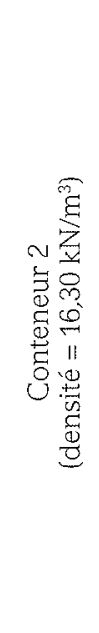 } & 1 & 1,9 & 60 \\
\hline & 2 & 2,8 & 88 \\
\hline & 3 & 4,1 & 116 \\
\hline & 4 & 7,7 & 177 \\
\hline & 5 & 12,5 & 234 \\
\hline & 6 & 10,7 & 275 \\
\hline & 7 & 22,4 & 413 \\
\hline & 8 & 35,1 & 475 \\
\hline & 9 & 35,1 & 475 \\
\hline
\end{tabular}




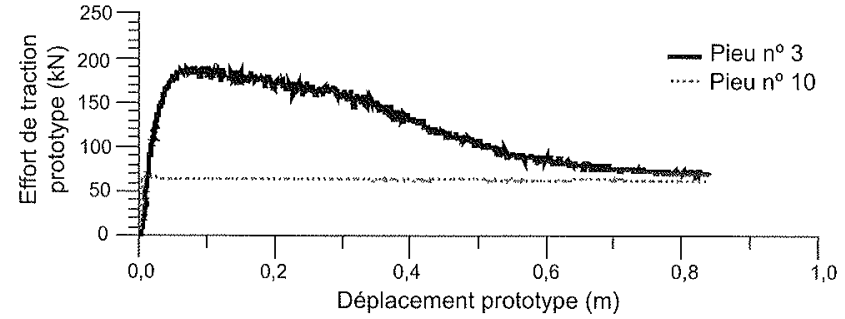

ric 10 Effort de traction $x$ déplacement des pieux modèles $n^{\circ} 3$ (avec hélice) et $n^{\circ} 10$ (sans hélice) installés dans le conteneur 2.

Tension force $\mathrm{x}$ clisplacement of the model piles $n^{\circ} 3$ (with helical plates) and $n^{\circ} 10$ (without helical plates) in the container 2.

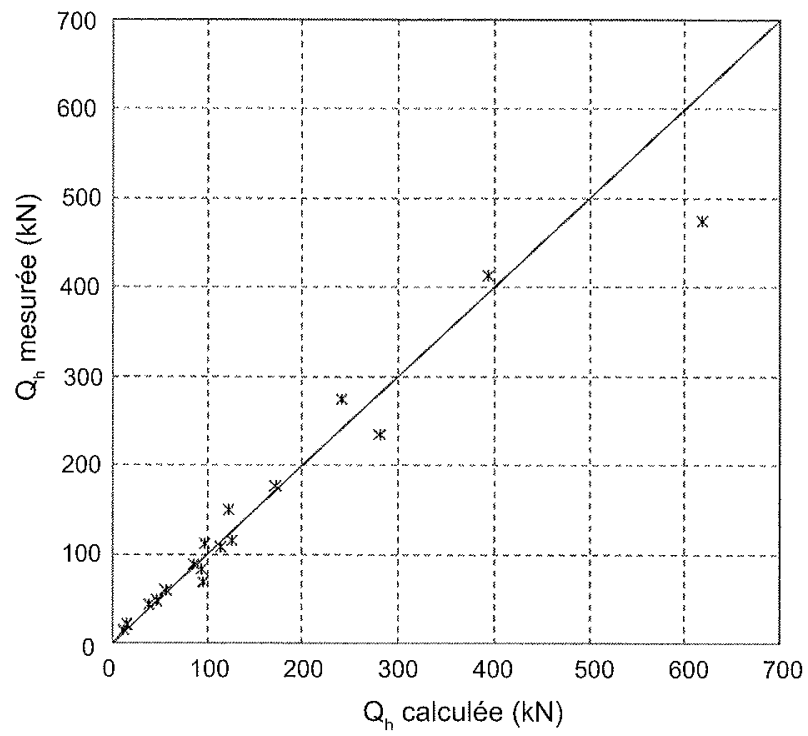

fG 11 Comparaisons entre les valeurs calculées et mesurées de la charge d'arrachement relative aux hélices $Q_{h}$ (en données prototypes).

Comparison of predicted and measured uplift helix bearing capacities $Q_{h}$ (prototype values).

\section{Comparaisons avec des essais sur pieux en vraie grandeur}

Tsuha (2007) a réalisé des essais d'arrachement (Fig. 12) sur quatre pieux hélicoïdaux (un pieu avec deux hélices et trois pieux avec trois hélices) mis en place dans un silt sableux moyennement compact. A Contagem (Brésil), la valeur $\mathrm{N}$ d'essais de pénétration standard (SPT) moyenne mesurée à la profondeur des hélices est de 25 coups pour une pénétration de $30 \mathrm{~cm}$. Les pieux sont de taille et de fiche semblables aux modèles 5 et 6 testés en centrifugeuse pour cette recherche (Tableau V).

La comparaison entre les résultats acquis des essais en centrifugeuse sur les pieux modèles 5 et 6 (Tableau I) et les résultats déduits des essais en vraie grandeur est présentée dans le tableau V. Bien que les deux groupes de pieux (modélisés en centrifugeuse et expérimentés en vraie grandeur) ont été mis en place dans plusieurs massifs et à des profondeurs différentes, les résultats (sur pieu avec le même nombre d'hélices) sont du même ordre de grandeur.

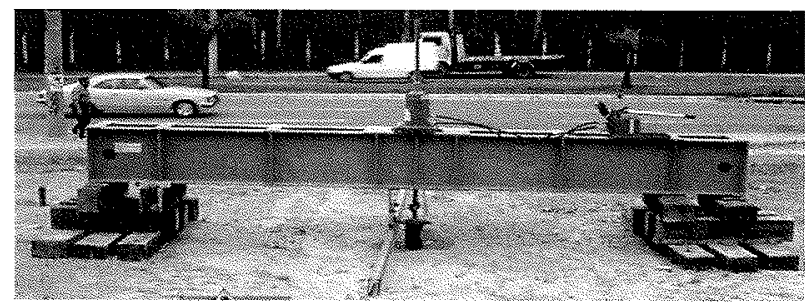

FG. 12 Essais d'arrachement sur pieu hélicoïdal en vraie grandeur.

Uplift test of a full-scale helical pile.

TABLAuv Comparaison entre les résultats des essais en centrifugeuse (convertis en prototype) et des essais en vraie grandeur.

Comparison between centrifuge tests results (prototype values) and full-scale tests results

\begin{tabular}{|c|c|c|c|c|c|}
\hline Essais diarraternent & Pleti & Nembre a nelices & $\begin{array}{l}\text { Diametre helice } \\
\text { (mm) }\end{array}$ & $\begin{array}{l}\text { Prie } \\
\text { (n) }\end{array}$ & $\begin{array}{l}\text { Charge d arrachement } \\
\text { retative a t helices } 9 \text {. kN }\end{array}$ \\
\hline \multirow{2}{*}{$\begin{array}{c}\text { Centrifugeuse } \\
(\text { Conteneur } 1) \\
\text { sable } I_{D}=56 \%\end{array}$} & 5 & 2 & 326 et 326 & 4,6 & 83 \\
\hline & 6 & 3 & 326,326 et 326 & 4,6 & 112 \\
\hline \multirow{2}{*}{$\begin{array}{l}\text { Centrifugeuse } \\
\text { (Conteneur 2) } \\
\text { sable } \mathrm{I}_{\mathrm{D}}=85 \%\end{array}$} & 5 & 2 & 326,326 et 326 & 4,6 & 234 \\
\hline & 6 & 3 & 326,326 et 326 & 4,6 & 275 \\
\hline \multirow{4}{*}{$\begin{array}{c}\text { Vraie grandeur } \\
\text { (silt sableux moyennement } \\
\text { compact) }\end{array}$} & $1 \mathrm{~V}$ & 2 & 305 et 356 & 5,6 & 180 \\
\hline & $2 \mathrm{~V}$ & 3 & 254,305 et 305 & 5,5 & 168 \\
\hline & $3 \mathrm{~V}$ & 3 & 254,305 et 305 & 5,9 & 260 \\
\hline & $4 \mathrm{~V}$ & 3 & 254,305 et 356 & 5,9 & 227 \\
\hline
\end{tabular}




\section{Essai d'arrachement dans du sable avec des couches colorées}

Il était intéressant de visualiser les lignes de rupture autour des hélices après essais d'arrachement. Cette approche qualitative doit permettre de confirmer le type de comportement.

Pendant la phase de remplissage du conteneur, les pieux modèles sont maintenus verticalement dans le conteneur par des fils en nylon de très faible épaisseur de façon à assurer une pré-fiche. Le sable de Fontainebleau (identique à celui utilisé pour les essais en centrifugeuse avec $\mathrm{I}_{\mathrm{D}}=85 \%$ ), est mis en place par séquences consécutives de pluviation interrompues après chaque dépôt de $20 \mathrm{~mm}$ d'épaisseur. La surface est alors nivelée et une fine couche de sable coloré est saupoudrée. La séquence suivante est alors enchaînée jusqu'au remplissage final du conteneur. Pour ces essais, seuls les pieux avec hélices de diamètre $20 \mathrm{~mm}$ (pieux 7, 8 et 9 de la figure 5) ont été utilisés pour s'assurer d'une meilleure sensibilité des phénomènes à observer.

Ces essais d'arrachement ont été réalisés sur des pieux installés à $1 \mathrm{~g}$ dans un massif de sable non saturé. Après mise en place par rotation du pieu hélice, l'essai d'arrachement est pratiqué en limitant volontairement le déplacement vertical à quelques millimètres; les pieux sont alors maintenus dans cette position pendant la saturation du massif de sable par la base. Après un essorage lent, il est possible de découper verticalement le massif sans perturber sa tenue; le découpage laisse apparaître les couches colorées déformées par les ruptures locales.

On observe sur la figure 13 que la couche de sable colorée (1) directement située au dessus de l'hélice est affectée par celle-ci, mais ni la couche intermédiaire (2) ni la troisième (3) ne sont affectées. Ces observations confirment les travaux de Adams et Klym (1972), qui ont affirmé que, lors de l'arrachement d'un pieu hélicoïdal multi-hélices, chaque hélice se comporte de façon indépendante à la condition que l'espacement vertical entre deux hélices soit au moins égal à deux fois le diamètre de l'hélice inférieure. En plus, ce résultat qualitatif valide également l'hypothèse retenue (équation 2) pour le modèle proposé, où la charge d'arrachement relative aux hélices $Q_{h}$ est la somme des capacités individuelles de chaque hélice.

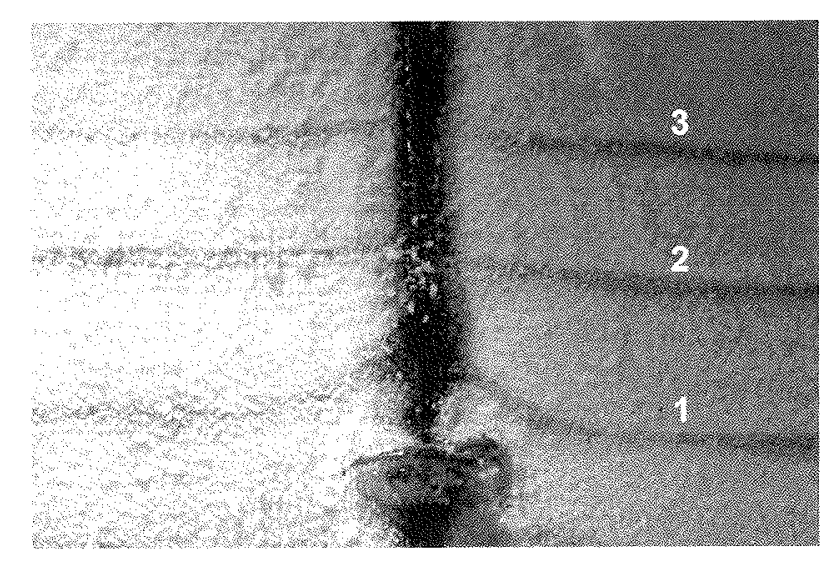

16. 13. Photographie dumassif de couches colorées découpé après l'essai d'arrachement. Photography of the sand sample cut after pullout test.

\section{Conclusion}

Cette approche expérimentale réalisée en centrifugeuse a fourni des données essentielles pour la vérification d'une méthode de calcul de la charge d'arrachement relative aux hélices des pieux hélicoïdaux dans du sable à partir du couple mesuré en fin d'installation. La comparaison entre valeurs mesurées et calculées valide la relation théorique proposée qui permet une excellente prévision de la résistance des pieux.

La relation entre le couple de fonçage et la résistance à l'arrachement mobilisés par les hélices s'avère valide quel que soit le nombre d'hélices (jusqu'à 3 avec une distance minimale entre les hélices égale à 3D) et leur diamètre (entre 21 et $44 \mathrm{~cm}$ ).

Les essais d'arrachement des pieux modèles réalisés clans le conteneur préparé avec des couches intermédiaires de sable coloré ont montré que la zone d'influence de l'hélice se limite à une distance inférieure ou égale à deux fois le diamètre l’hélice.

L'ordre de grandeur des résultats obtenus par la modélisation physique en centrifugeuse est comparable aux essais réalisés sur pieux hélicoïdaux à l'échelle réelle.

\section{Bibliographie}

Adams J.I., Klym T.W. - A study of anchors for transmission tower foundations. Canadian Geotechnical Journal, vol. 9, $n^{\circ} 1,1972$, p. 89-104.

Corté J.-F. - L'essor de la modélisation en centrifugeuse en géotechnique. Revue française de géotechnique, $n^{\circ} 48,1989$, p. 7-13

Garnier J., Derkx F., Cottineau L.M. Rault G. - Études géotechnicues sur modéles réduits centrifugés - Évolution des matériels et techniques expérimentales. Bulletin des laboratoires des ponts et chaussées, $n^{\circ} 223,1999$, p. 27-50.

Garnier J., Gaudin C., Springman S.M., Culligan P.J., Goodings D., Konig D., Kutter B., Phillips R., Randolph M.F., Thorel L. - Catalogue of scaling laws and similitude questions in geotechnical centrifuge modelling. Int. J. Physical Modelling in Geotechnics, vol. $7, n^{\circ} 3$, 2007, p. 1-24.

Ghaly A., Hanna A. - Experimental and theoretical studies on installation torque of screw anchors. Canadian Geotechnical Joumnal, vol. 28, n³, 1991, p. 353-364.

Ghaly A., Hanna A., Hanna M. - Installation torque of screw anchors in sand. Soils and Foundations, vol. 31, n 2, 1991, p. 77-92.

Narasimha Rao, S., Prasad, M.D., Shetty, M.D., Joshi, V.V. - Uplift capacity of screw pile anchors. Geotechnical Engineering, vol. 20, n² 2, 1989, p.139-159.

Perko H.A. - Energy method for predicting the installation torque of helical foun- dations and anchors. New Technological and Design Developments in Deep Foundation Technologies, ASCE, 2000, p. 342-352.

Thorel L., Rault G., Garnier J., Murillo C., Gaudicheau P., Néel A., Favraud C. Mesures en macrogravité sur modèles réduits d'ouvrages. Bulletin de liaison des Ponts et Chaussées, $n^{\circ}$ 272-273, spécial Métrologie, 2008, p. 93-131.

Tsuha C.H.C. - Theoretical model to control on site the uplift capacity of helical screw piles embedded in sandy soil. Ph. D. thesis, Department of Geotechnics, São Carlos School of Engineering, University of Säo Paulo, Brazil, 2007. 